\title{
Uber Hexokinase-Reaktion und Atmung in der lebenden Zelle
}

\author{
Von Lutz Kiesow \\ Aus dem Physiologisch-chemischen Institut der Freien Universität Berlin \\ (Direktor: Professor Dr. Dr. ERnst Schütte) \\ (Z. Naturforschg. 15 b, 171-174 [1960] ; eingegangen am 9. Dezember 1959)
}

\begin{abstract}
Man kann an der intakten, lebenden Zelle mit Hilfe von 2-Desoxyglucose messen, wie schnell die Hexokinase-Reaktion bei einer bestimmten Zellatmung abläuft.
\end{abstract}

Gibt man zu einer Salzlösung, in der mit Sauerstoff gesättigte Kulturhefezellen * oder Krebszellen suspendiert sind, 2-Desoxyglucose, so nimmt die Konzentration dieses Zuckers in der Lösung so lange $a b$, bis sie einen stationären Zustand erreicht. Wenn man nun den Sauerstoff der Gasphase durch Argon * ersetzt, dann erscheint die Desoxyglucose wieder in der Lösung. Das ist ein Versuch, den man an ein und derselben Zellsuspension beliebig oft wiederholen kann ${ }^{1}$, und die Chemie der Vorgänge, die dabei in den Zellen ablaufen, zeigt Abb. 1.

Es ist die Photographie eines Chromatogramms wäßriger Hitzeextrakte der einzelnen Phasen des eben beschriebenen Versuches. Zu den sauerstoffgesättigten Kulturhefezellen in Wasser wurden $200 \mathrm{mg}$-\% 2-Desoxyglucose zugesetzt und sofort danach ein kleiner Teil der Suspension 5 min im siedenden Wasserbad inaktiviert und extrahiert. Vom klar zentrifugierten Überstand wurden $0,05 \mathrm{~cm}^{3}$ auf das Papier aufgetragen (Startpunkt 1). Die Suspension wird im Thermostaten bei $20^{\circ} \mathrm{C} 90 \mathrm{~min}$ lang weitergeschüttelt und mit einem Teil von ihr die gleiche Extraktion vorgenommen (Startpunkt 2). Dann wird der Sauerstoff durch Argon ersetzt. Nach weiteren $90 \mathrm{~min}$ in Argon erhält man die Lösung des 3. Startpunktes. Das entwickelte und angefärbte Chromatogramm zeigt in seiner oberen Fleckenreihe, wie sich bei diesem Versuch die Desoxyglucose und in seiner unteren wie das aus ihr in den Zellen entstandene 2-Desoxyglucose-6-phosphat verhält. In Sauerstoff verschwindet der freie Zucker und in Argon erscheint er wieder. In Sauerstoff entsteht der 6-Phosphorsäureester dieses Zuckers und in Argon verschwindet er wieder. Übertragen auf die Veränderungen der Zuckerkonzentration in dem Sus-

* Herr Dr. von Lacrorx, von der Norddeutschen Hefeindustrie, Hamburg, hat mir die Hefe zur Verfügung gestellt und Herr Dr. Franz, von der Osram Gesellschaft, das sauerstoff-freie Argon. Beiden Herren möchte ich an dieser Stelle dafür vielmals danken.

1 L. Kiesow, Z. Naturforschg. 14 b, 492 [1959]. pensionsmedium der Zellen heißt das, der Zucker nimmt aerob ab, weil ihn die Hexokinase der Zellen phosphoryliert, die Abnahme wird stationär, weil die Spaltung des Zuckers durch Phosphatase seiner Phosphorylierung entgegenwirkt, und er erscheint

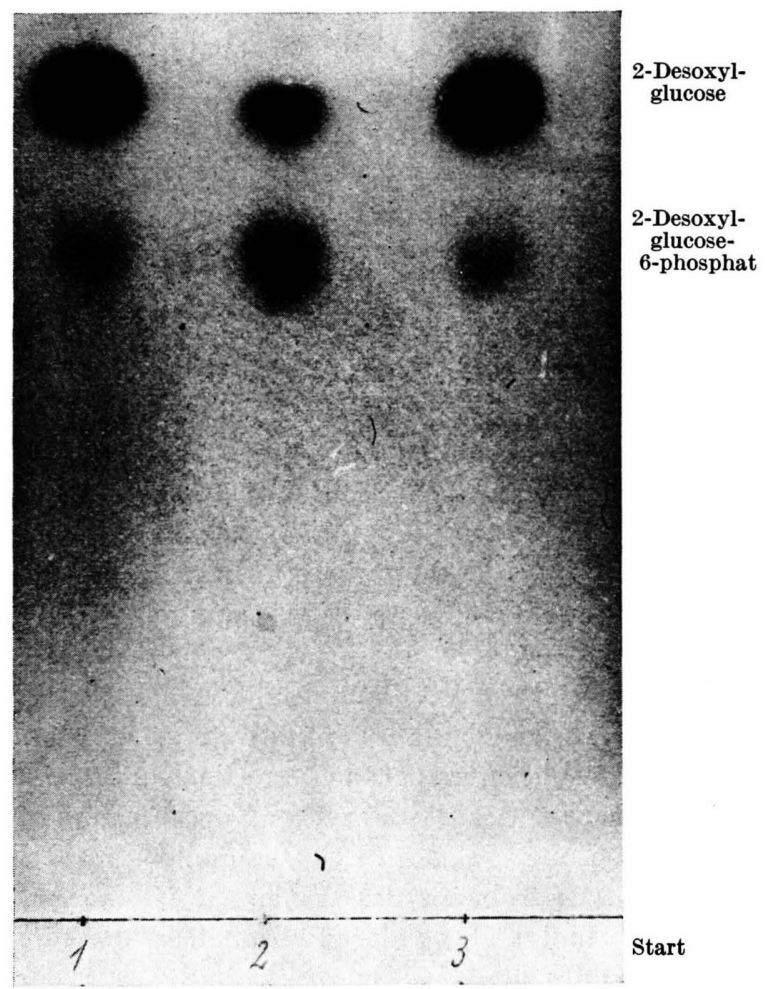

Abb. 1. Chromatogramm von in der Hitze extrahierten Zellen, die aerob 2-Desoxyglucose phosphorylieren und aufnehmen und die sie anaerob dephosphorylieren und abgeben. Je $200 \mathrm{~mm}^{3}$ Kulturhefezellen waren in $1 \mathrm{~cm}^{3}$ Wasser suspendiert. Zur Zeit $t=0$ wurden $200 \mathrm{mg}-\%$ 2-Desoxyglucose zugesetzt, und ein Teil der Suspension in der Siedehitze extrahiert (1). 90 min wird in $\mathrm{O}_{2}$ bei $20^{\circ}$ geschüttelt (2) und anschließend $90 \mathrm{~min}$ in Argon (3). Die Flecken steigen auf Schleicher \& Schüll-Papier $2043 \mathrm{~b}$ bei $+10^{\circ} \mathrm{C}$ in Isopropanol : 20\% Trichloressigsäure in Wasser : Wasser : 25-proz. Ammoniak = $35: 10: 4,5: 0,1548 \mathrm{Stdn}$. lang auf. Das trokkene Papier wird mit einem Gemisch gleicher Teile 5-proz. Perchlorsäure und 1-proz. Ammoniummolybdat in Wasser besprüht und 5 min auf $105^{\circ}$ erwärmt. 
anaerob wieder, weil die Zellen nicht mehr atmen und nur noch die phosphatatische Spaltung wirkt. Der stationäre Zustand entsteht also dadurch, daß sich die Reaktionsgeschwindigkeiten zweier Reaktionen überlagern und da $\beta$ das Reaktionsprodukt der einen das Substrat der anderen ist, und es muß weiter die Reaktionsgeschwindigkeit $v$ der Hexokinase genau so groß sein wie die Anfangsgeschwindigkeit $v_{0}$ der Phosphatase. Unterbindet man die Phosphorylierung des Zuckers dadurch, daß man die Zellen anaerob macht und somit der Hexokinase die ATP entzieht, so ist die Geschwindigkeit, mit der der Desoxyzucker wieder in der Lösung erscheint, gleich $\operatorname{dem} v_{0}$ der Phosphatase-Reaktion.

(Eine ähnliche Überlegung hat meines Wissens LyNEN $^{2,3}$ als erster für das Orthophosphat angestellt, als er die Entstehung von Orthophosphat in Trichloressigsäure-Extrakten von Hefezellen gemessen hat, deren Atmung und Gärung durch 0,1-m. HCN gehemmt waren. Die Anfangsgeschwindigkeit der Dephosphorylierungen wurde dabei gleich der Geschwindigkeit der Phosphorylierung durch Atmung und Gärung gesetzt.)

Man hat mit anderen Worten die Möglichkeit auf diesem Wege die Geschwindigkeit der Hexokinase-Reaktion in der lebenden Zelle zu messen, ohne die Zelle abzutöten oder zu extrahieren. Da man mit der manometrischen Methode unter den gleichen Bedingungen die Atmung messen kann, ist aus beiden Größen leicht zu berechnen, wieviel ATP unter den jeweiligen Versuchsbedingungen die Atmung mindestens bildet und wieviel davon der Hexokinase mindestens zu Verfügung steht.

Die Abb. 2 zeigt die quantitativen Zusammenhänge eines solchen Experimentes. Die Kurve zeigt die Veränderung der Desoxyzucker-Konzentration in Phosphat-Lösung, in der Kulturhefezellen suspendiert sind. Der Tangens des Winkels $\varphi$ ist die Anfangsgeschwindigkeit $v_{0}$ der Phosphatase-Reaktion und somit auch die Reaktionsgeschwindigkeit $v$ der Hexokinase. Die Geschwindigkeit der Zucker-Aufnahme kann man zur Berechnung von der Hexokinase nicht verwenden, weil die Atmung und damit die ATP-Bildung in den Zellen noch nicht stationär ist.

\section{Methodische Vorbemerkungen und Definitionen}

Bei einem vollständigen Versuch dieser Art wird die Zellatmung manometrisch gemessen und die

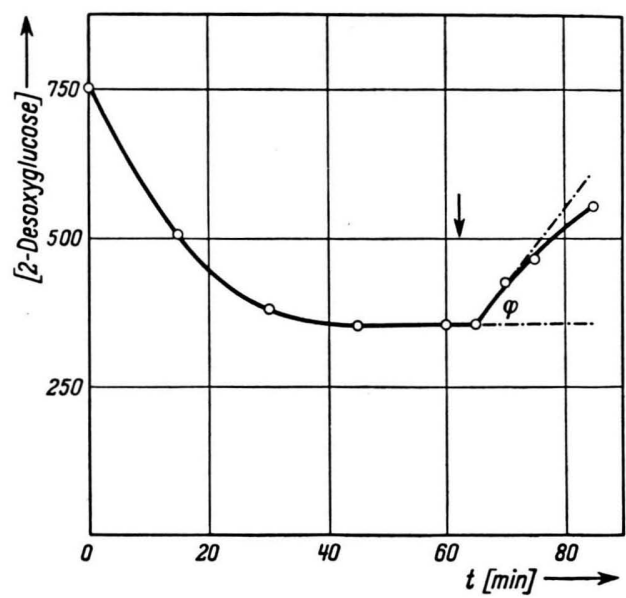

Abb. 2. Aerobe und anaerobe Änderungen der 2-Desoxyglucose-Konzentration in $m / 20-\mathrm{KH}_{2} \mathrm{PO}_{4}$. Sie enthielt in $1 \mathrm{~cm}^{3}$ $165 \mathrm{~mm}^{3}$ Kulturhefezellen. Die Ordinate zeigt die Desoxyglucose in $\gamma$, die in $0,5 \mathrm{~cm}^{3}$ Suspensionsmedium enthalten ist. Diese $0,5 \mathrm{~cm}^{3}$ Medium entsprechen $99 \mathrm{~mm}^{3}$ oder $25,7 \mathrm{mg}$ Trockengewicht Kulturhefezellen. Die Abszisse gibt die Zeit in Minuten. Zur Zeit der Markierung wird der Sauerstoff durch Argon ersetzt. Der $\operatorname{tg} \varphi$ ist $v_{0}=13,4 \gamma /$ min oder als $Q$ Desoxyglucose $=4,3$.

Konzentration der 2-Desoxyglucose-Lösung in $\mathrm{Ab}$ hängigkeit von der Zeit im Suspensionsmedium bestimmt. Dabei werden die Zellen zuerst 60 min lang mit Sauerstoff gesättigt (von etwa der 45. min an ist die 2-Desoxyglucose-Aufnahme bei $20^{\circ}$ stationär. $\mathrm{Zu}$ diesem Zeitpunkt wird auch die Atmung gemessen). Dann leitet man Argon ein und nach weiteren 20-30 min ist der Versuch beendet, da nur die Anfangsphase des Wiedererscheinens des Zuckers im Medium von Interesse ist. In den erforderlichen Zeitabständen werden $2-3 \mathrm{~cm}^{3}$ des Versuchsansatzes auf Schottschen $\mathrm{G}_{4}$-Glasfritten schnell von den Zellen befreit und in je $0,5 \mathrm{~cm}^{3}$ des zellfreien Mediums der Desoxyglucose-Gehalt gemessen. Das kann mit der quantitativen StoLLschen Methode ${ }^{1}$ oder auch mit Glucoseoxydase und Peroxydase fermentativ erfolgen. Beide analytischen Methoden liefern Werte der Lichtschwächung, die mit Hilfe der molaren Extinktionen in $\gamma$ 2-Desoxyglucose umzurechnen sind. So kommt man zu graphischen Darstellungen wie die der Abb. 2. Aus ihr entnimmt man als Anfangsgeschwindigkeit der Spaltung des

2 F. Lynen u. R. Koenigsberger, Liebigs Ann. Chem. 573, 60 [1951].

3 F. Lynen, in: Proc. of the III. International Congress of Biochemistry, Brüssels 1955, Academic Press, New York 1956, S. 294. 
Desoxyglucose-6-phosphates bei $20^{\circ}$ :

$$
\begin{aligned}
v_{0}=\operatorname{tg} \varphi & =\frac{\Delta c}{\Delta t} \operatorname{oder} \frac{\Delta \ln \left(i_{0} / i\right)}{\Delta t}, \\
{\left[v_{0}\right] } & =\left[\frac{\gamma 2 \text {-Desoxyglucose }}{\min }\right],
\end{aligned}
$$

Aus der Einleitung ging hervor:

$$
v_{0}=v_{\text {Hexokinase }} \text {. }
$$

Um eine Beziehung zu W a r b u r g s Quotienten der Atmung, dem $Q_{\mathrm{O}_{2}}$ bei $20^{\circ} \mathrm{C}$, dessen Dimension

$$
\frac{\mathrm{mm}^{3} \mathrm{O}_{2}}{\text { mgTrockengewicht } \cdot \text { Stunde }}
$$

ist, zu bekommen, kann man $v_{0}$ folgendermaßen umrechnen und beziehen:

$$
Q_{\text {Desoxyglucose }}=\frac{v_{0}}{\mathrm{mgTrockengewicht} \cdot 60 \mathrm{~min}},
$$

wobei die Größe $v_{0}$ dann die Dimension

$$
\left[v_{0}\right]=\left[\frac{\Delta c}{\Delta t}\right]=\left[\frac{\mathrm{mm}^{3} \text { Desoxyglucose }}{\min }\right]
$$

hat. Somit ist die Dimension von

$$
\left[Q_{\text {Desoxyglucose }}\right]=\left[\frac{\mathrm{mm}^{3} \text { Desoxyglucose }}{\mathrm{mg}_{\text {Trockengewicht }} \cdot \text { Stunde }}\right]
$$

\section{Hexokinase-Reaktion bei endogener Atmung}

Wenn man Kulturhefezellen in $m / 20-\mathrm{KH}_{2} \mathrm{PO}_{4}$ suspendiert und der Suspension $200 \mathrm{mg}$-\% 2-Desoxyglucose zusetzt, so findet man mit der eben beschriebenen Methode die Werte der Tab. 1 und mit Hilfe der Manometrie die der Tab. 2.

\begin{tabular}{|c|c|}
\hline$v_{0}$ & $Q$ Desoxyglucose \\
\hline mg Trockengewicht $\cdot 60 \mathrm{~min}$ & \\
\hline 32,9 & 4,5 \\
29,3 & 4,3 \\
41,0 & 4,0 \\
26,5 & 5,6 \\
27,2 & 3,6 \\
35,6 & 3,7 \\
im Mittel: & 4,9 \\
\hline
\end{tabular}

Tab. 1. Anfangsgeschwindigkeiten der Spaltung des Desoxyglucose-6-phosphates und Quotienten der Desoxyglucose-

Phosphorylierung durch Kulturhefe in $m / 20-\mathrm{KH}_{2} \mathrm{PO}_{4}$ bei $20{ }^{\circ} \mathrm{C}$.

Da die Zellen unter diesen Bedingungen nur endogenes Substrat veratmen, sagt der $Q_{\text {Desoxyglucose }}$ als $\mathrm{Ma}$ für die Geschwindigkeit der Hexokinase-Reaktion, wieviel ATP dabei am Ort der Hexokinase reagiert und somit auch wieviel ATP die Atmung min-

\begin{tabular}{|c|c|}
\hline$Q \mathrm{o}_{2}\left[\frac{\mathrm{mm}^{3} \mathrm{O}_{2}}{\mathrm{mg} \text { Trockengewicht } \cdot \text { Stunde }}\right]$ \\
\hline \\
$-2,43$ \\
$-2,36$ \\
$-2,36$ \\
$-2,54$ \\
$-2,45$ \\
$-2,45$ \\
& $-2,49$ \\
& $-2,48$ \\
\hline im Mittel:
\end{tabular}

Tab. 2. Die Quotienten der Atmung von Kulturhefe in $m / 20-\mathrm{KH}_{2} \mathrm{PO}_{4}$ mit $200 \mathrm{mg}-\%$ 2-Desoxyglucose bei $20^{\circ} \mathrm{C}$. Manometrie: Kastenförmige Gefäße mit zentraler Wanne ${ }^{4}$, die $0,2 \mathrm{~cm}^{3}$ 2-n. $\mathrm{KOH}$ enthielt. Im Hauptraum sind $3 \mathrm{~cm}^{3}$ Zellsuspension. $\nu=200 / \mathrm{min} ; \varepsilon=1,4 \mathrm{~cm}$. Für die Berechnung wird die Atmung verwendet, die man 45-60 min nach dem Sättigen mit Sauerstoff mißt.

destens bilden muß. Benutzt man den für die sog. oxydative Phosphorylierung der Mitochondrien eingeführten $\mathrm{P} / \mathrm{O}$-Quotienten, so wird hier an der lebenden Zelle, ohne daß die Zelle dazu abgetötet, aufgeschlossen oder in irgendeiner anderen Weise verändert werden muß, ein Quotient von

$$
\frac{Q_{\text {Desoxyglucose }}}{2 Q_{0_{2}}}=\frac{4,4}{2 \cdot 2,5}=0,88
$$

gemessen.

Die Ermittlung der Fehler der beiden Größen dieses Quotienten sowie die Berücksichtigung der Fortpflanzung dieser Fehler zeigen, daß der Wert auf $6 \%$ genau ist.

\section{Hexokinase-Reaktion bei Alkohol-Atmung}

Der Zusatz von Äthylalkohol zu der Suspension von Kulturhefe in $m / 20-\mathrm{KH}_{2} \mathrm{PO}_{4}$ mit $200 \mathrm{mg}-\%$ 2-Desoxyglucose bewirkt eine Erhöhung der Zellatmung auf einen $Q_{\mathrm{O}_{2}}$ von -20 , wie die Tab. 4

\begin{tabular}{|c|c|}
\hline$v_{0}$ & $Q$ Desoxyglucose \\
\hline mg Trockengewicht $\cdot 60 \mathrm{~min}$ & \\
\hline 40,5 & 5,5 \\
51,7 & 7,1 \\
49,4 & 6,8 \\
53,8 & 7,4 \\
61,2 & 8,4 \\
57,8 & 7,9 \\
\hline im Mittel: & 7,2 \\
\hline
\end{tabular}

Tab. 3. Anfangsgeschwindigkeiten der Spaltung von Desoxyglucose-6-phosphat und die Quotienten der DesoxyglucosePhosphorylierung durch Kulturhefe in $m / 20-\mathrm{KH}_{2} \mathrm{PO}_{4}$ mit $2 \%$ Âthylalkohol. 


\begin{tabular}{|c|c|}
\hline $\mathrm{oo}_{2}\left[\frac{\mathrm{mm}^{3} \mathrm{O}_{2}}{\mathrm{mg} \text { Trockengewicht } \cdot \text { Stunde }}\right]$ \\
\hline \\
-21 \\
-22 \\
-16 \\
-19 \\
-18 \\
-24 \\
-23 \\
& -20 \\
\hline im Mittel: & \\
\hline
\end{tabular}

Tab. 4. Atmung von Kulturhefe in $m / 20-\mathrm{KH}_{2} \mathrm{PO}_{4}$ mit $2 \%$ Äthylalkohol und $200 \mathrm{mg}-\%$ 2-Desoxyglucose bei $20^{\circ} \mathrm{C}$. Manometrie wie in Tab. 2.

zeigt, d. h. die Atmung ist gegenüber der endogenen Atmung auf das 8-fache angestiegen.
Die Phosphorylierung des 2-Desoxyzuckers ist dabei, wie der $Q_{\text {Desoxyglucose }}$ in Tab. 3 zeigt, zwar auch schneller geworden, aber am Ort der Hexokinase reagiert nur 7,2/4,4=1,6-mal mehr ATP als bei der endogenen Atmung. Das geht auch aus dem Quotienten:

$$
\frac{Q_{\text {Desoxyglucose }}}{2 Q_{\mathrm{O}_{2}}}=\frac{7,2}{40}=0,18
$$

hervor, demzufolge bei der Veratmung von Alkohol rund $1 / 5$ der ATP am Ort der Hexokinase mit dem Zucker zur Reaktion kommen, wenn man die ATPBildung bei endogener Atmung zugrunde legt.

Frl. Maria Schrader und Frl. Liselotte Petersen haben mir bei den Versuchen geholfen. Ich möchte Ihnen dafür vielmals danken.

4 O. WarbUrG u. G. KripPahl, Z. Naturforschg. 13 b, 434 [1958].

\title{
Über Gärung und Chinon im Zellstoffwechsel
}

\author{
Von Lutz Kiesow \\ Aus dem Physiologisch-chemischen Institut der Freien Universität Berlin \\ (Direktor: Professor Dr. Dr. ERnst Schürte) \\ (Z. Naturforschg. 15 b, 174-179 [1960]; eingegangen am 11. Dezember 1959)
}

\begin{abstract}
Bei der Gärungshemmung durch Vitamin $\mathrm{K}_{3}$ kann Ferricyanid den Sauerstoff ersetzen. Das Ferricyanid wird dabei reduziert, weil es mit dem Hydrochinon, das in der Zelle entstanden ist, reagiert.

2.4-Dinitrophenol und Blausäure hemmen die Chinon-Reduktion. Die Gärungshemmung durch Chinon geht mit einer Hemmung der Hexokinase-Aktivität der intakten Zelle einher.
\end{abstract}

Wie vor kurzem mitgeteilt wurde ${ }^{1}$, kann man das Wachstum und die Gärung von Kulturhefe gleich stark durch 2-Methyl-1.4-naphthochinon hemmen. In diesem Zusammenhang wurde darauf hingewiesen, daß Sauerstoff für die Gärungshemmung durch das Chinon notwendig ist. Seitdem habe ich zwei Beobachtungen gemacht, die zu neuen Experimenten Anlaß geben. Danach kann Gärungshemmung durch Chinon auch anaerob zustande kommen, wenn die Zelldichte gering ist, und weiter kann man den Sauerstoff bei der Chinonhemmung der Gärung durch Kaliumferricyanid ersetzen.

Besonders die letztere Beobachtung hat zahlreiche neue Versuche möglich gemacht, von denen die vorliegende Arbeit einen Teil wiedergibt *.

1 L. Kiesow, Z. Naturforschg. 14 b, 490 [1959].

* Einige der hier beschriebenen Versuche wurden mit anderen, die noch nicht veröffentlicht sind, auf der Tagung für Physiologische Chemie am 23.-26. Sept. 1959 in Berlin vorgetragen.

* 2-Methyl-1.4-naphthochinon wird im folgenden nur kurz als „Chinon“ bezeichnet.

\section{Anaerobe Chinonwirkung und Zelldichte}

Wenn man die Gärung von Kulturhefe, die in Phosphat-Glucose suspendiert und mit Argon gesättigt ist, manometrisch mißt, findet man eine strenge Proportionalität zwischen $\mathrm{CO}_{2}$-Entwicklung und Zellmenge. Gibt man nun aus den Birnen der Manometriegefäße gleiche Mengen 2-Methyl-1.4-naphthochinon $^{* *}$ in die Haupträume, so kann starke Gärungshemmung bei sehr geringer Zelldichte und geringe oder gar keine Gärungshemmung bei größerer Zelldichte beobachtet werden (Abb. 1). Um dieses Ergebnis zu verstehen, haben wir die Zellen in den Manometriegefäßen unter Ausschluß des Sauerstoffs 5 Min. auf $100^{\circ}$ im Wasserbad erwärmt und sie gleichzeitig extrahiert. Ursula Schmitz hat die UVSpektren dieser Extrakte aufgenommen und dabei alle Übergänge zwischen reinem Hydrochinon- und reinem Chinon-Spektrum gefunden. Das erste lag bei hoher, das zweite bei niedriger Zelldichte vor. 